\title{
Outage Thresholds of LDPC Codes over Nonergodic Block-Fading Channels
}

\author{
Iryna Andriyanova \\ ETIS group \\ ENSEA/UCP/CNRS-UMR8051 \\ 95014 Cergy-Pontoise, France \\ iryna.andriyanova@ensea.fr
}

\author{
Ezio Biglieri \\ WISER S.r.1. \\ Via Fiume 23 \\ 57123 Livorno, Italy \\ e.biglieri@ieee.org
}

\author{
Joseph J. Boutros \\ Elec. Eng. Department \\ Texas A\&M University at Qatar \\ 23874, Doha, Qatar \\ boutros@ieee.org
}

\begin{abstract}
This paper ${ }^{1}$ derives approximations allowing the estimation of outage probability for standard irregular LDPC codes and full-diversity Root-LDPC codes used over nonergodic block-fading channels. Two separate approaches are discussed: a numerical approximation, obtained by curve fitting, for both code ensembles, and an analytical approximation for Root-LDPC codes, obtained under the assumption that the slope of the iterative threshold curve of a given code ensemble matches the slope of the outage capacity curve in the high-SNR regime.
\end{abstract}

\section{INTRODUCTION}

The nonergodic block-fading model is appropriate for a wide variety of wireless communication systems, for example using multiple antennas or retransmission schemes. The design of sparse-graph codes maximizing the transmit diversity for these channels has been first addressed in [1], where the authors proposed a new code family, the Root-LDPC codes, which attains the maximal theoretical diversity 2 over a blockfading channel with two independent fading realizations per coded data packet. In this paper we focus on this block-fading channel, and study how the iterative-decoding threshold depends on the two fading gains parametrizing the channel. Note that the computation of the iterative-decoding threshold for various fading coefficients is the main part of the computation of the outage probability, attainable for a given code ensemble. Note also that by now the case of ergodic channels (i.e. with one fading realization per coded data packet) is well handled; the iterative decoding threshold is estimated by means of the density evolution under semi-Gaussian approximation [2]. In the case of nonergodic channels the Gaussian approximation is not valid anymore, and, even if the density evolution can still be performed, it is done by direct computer simulations. The calculations made in this paper enable us to estimate the outage probability achievable with a given code ensemble without resorting to extensive, time-consuming simulations. Previous results [2] along this line allow to find only one point of the threshold curve- the one corresponding to equal fading gains-which corresponds to an ergodic block-fading channel [3].

Our channel model here is the same as in [1], where one half of the coded data block is affected by a Rayleigh-distributed

\footnotetext{
${ }^{1}$ This work was supported by the European FP7 ICT-STREP DAVINCI project under contract INFSO-ICT-216203.
}

fading gain $\alpha_{1}$, and the other half by a similarly distributed, independent gain $\alpha_{2}$. Two different types of code ensembles will be examined: standard random LDPC codes and RootLDPC codes. We hasten to recall that, while the random LDPC codes diversity is only 1 , Root-LDPC codes achieve transmit diversity 2 , and hence their error probability after decoding decreases as $\mathrm{SNR}^{-2}$.

This paper is organized as follows. The relevant definitions of code ensembles are given in Section II. Transmission model and threshold curves are introduced in Section III. Numerical and analytical approximations of the threshold curve are derived in Sections IV and V, respectively. Section VI concludes the paper.

\section{Two LDPC Code EnsEmbles: DeFINITION}

We examine two different code families: random LDPC codes and Root-LDPC codes, the latter being a family specially designed for transmission over block-fading channels with two subblocks. Specifically, our code ensembles are defined as follows.

1) Irregular LDPC ensemble: A $(\lambda, \rho)$ LDPC ensemble is a set of binary codes whose parity-check matrix contains a fraction $\lambda_{i}$ of columns with $i$ nonzero entries, $i \in$ $\left\{i_{1}, i_{2}, \ldots, i_{\max }\right\}$, and a fraction $\rho_{j}$ of rows with $j$ nonzero entries, $j \in\left\{j_{1}, j_{2}, \ldots, j_{\max }\right\}$. The code ensemble is completely described by the polynomials

$$
\lambda(x) \triangleq \sum_{i} \lambda_{i} x^{i-1} \quad \text { and } \quad \rho(x) \triangleq \sum_{j} \rho_{j} x^{j-1}
$$

2) Root-LDPC ensemble: A $(\lambda, \rho)$ Root-LDPC ensemble is defined with the help of multinomials $\lambda_{\text {root }}(\underline{\mu}, \underline{x})$ and $\rho_{\text {root }}(\underline{\mu}, \underline{x})$ with $\underline{\mu} \triangleq\left(\mu_{1}, \mu_{2}\right)$ and $\underline{x} \triangleq\left(x_{1}, x_{2}, x_{3}, x_{4}, x_{5}, x_{6}\right)$ :

$$
\begin{aligned}
\lambda_{\text {root }}(\underline{\mu}, \underline{x})= & \frac{1}{2} \sum_{i}\left(\frac{\lambda_{i}}{i} \mu_{1} x_{1}^{i}+\frac{(i-1) \lambda_{i}}{i} \mu_{1} x_{2}^{i}+\lambda_{i} \mu_{1} x_{3}^{i}\right. \\
& \left.+\lambda_{i} \mu_{2} x_{4}^{i}+\frac{(i-1) \lambda_{i}}{i} \mu_{2} x_{5}^{i}+\frac{\lambda_{i}}{i} \mu_{2} x_{6}^{i}\right), \quad(1)
\end{aligned}
$$




$$
\begin{aligned}
\rho_{\text {root }}(\underline{\mu}, \underline{x})=\frac{1}{2} \sum_{i} \rho_{i}\left(x_{1} \sum_{j}\left(\begin{array}{l}
i \\
j
\end{array}\right) f_{e}^{j} x_{4}^{j} g_{e}^{i-j} x_{5}^{i-j}\right. \\
\left.+x_{6} \sum_{k}\left(\begin{array}{l}
i \\
k
\end{array}\right) f_{e}^{k} x_{3}^{k} g_{e}^{i-k} x_{2}^{i-k}\right) .
\end{aligned}
$$

Here the variables $\mu_{1}$ and $\mu_{2}$ correspond to the two fading gains, and the variables $x_{1}, x_{2}, \ldots, x_{6}$ to 6 edge types in the bipartite code graph. Note that $x_{3}$ and $x_{4}$ are related to "parity" edges, and the remaining variables to "information" edges, reflecting the fact that the structure of the root-code graph consists of four types of variable nodes (denoted $1 i, 1 p, 2 i$ and $2 p$ ) and two of check nodes (denoted $1 c$ and $2 c$ ). Permutations of edges within edge classes are chosen uniformly at random. Variable nodes $1 i$ and $2 i$ correspond to the information bits in a codeword, while variable nodes $1 p$ and $2 p$ correspond to the redundant (parity) bits. Also, $f_{e}$ (resp., $g_{e}$ ) denotes the probability that an edge connected to a given check node is a parity (resp., information) edge. Clearly, $f_{e}+g_{e}=1$. As discussed in detail in [1], this structure generates a code ensemble of rate $1 / 2$.

\section{TRANSMISSION OVER BLOCK-FADING CHANNELS: ITERATIVE THRESHOLD $\gamma^{*}$ VERSUS FADING GAINS}

In our model, a codeword is divided into two equal subblocks, transmitted over two Rayleigh fading channels with common signal-to-noise ratio $\mathrm{SNR}=\gamma$ and independent fading gains $\alpha_{1}$ and $\alpha_{2}$. The observation $y_{i}$ resulting from the transmission of binary symbol $x= \pm 1$ received from the $i$ th channel, $i=1,2$, has the form $y_{i}=\alpha_{i} x+z_{i}$, where $\alpha_{i} \in[0,+\infty)$, and $z_{i}$ are independent and Gaussian distributed, $z_{i} \sim \mathcal{N}\left(0, \sigma^{2}\right)$, with $\sigma^{2}=1 / \gamma$.

The received data block is decoded using a standard belief propagation algorithm. The asymptotic performance of a given code ensemble is described by the iterative decoding threshold $\gamma^{*}$ - the minimum SNR at which reliable transmission is possible, for fixed fading gains $\alpha_{1}$ and $\alpha_{2}$ and infinite code length. Let the function $\gamma^{*}\left(\alpha_{1}, \alpha_{2}\right)$ denote the iterative threshold as a function of the fading gains realizations. If one knew $\gamma^{*}\left(\alpha_{1}, \alpha_{2}\right)$ for a given code ensemble, the outage probability $P_{\text {out }}$ could be obtained by observing that, with $\alpha_{1}$ and $\alpha_{2}$ independent unit-mean Rayleigh random variables,

$$
P_{\text {out }}=\int_{\left(\alpha_{1}, \alpha_{2}\right) \in R^{*}} e^{-\alpha_{1}^{2}-\alpha_{2}^{2}} d \alpha_{1}^{2} d \alpha_{2}^{2},
$$

where $R^{*}$ denotes the region below the threshold curve $\gamma^{*}\left(\alpha_{1}, \alpha_{2}\right)$ (see [3] for illustration). In general, $\gamma^{*}\left(\alpha_{1}, \alpha_{2}\right)$ can be obtained by performing the density evolution for each point of the curve. In this paper we advocate two kinds of approximations for $\gamma^{*}\left(\alpha_{1}, \alpha_{2}\right)$ : one numerical and another obtained analytically.

Before listing our results, we state the following useful lemma:

\section{Lemma 1:}

$$
\gamma^{*}\left(\alpha_{1}, \alpha_{2}\right)=\frac{\gamma^{*}\left(\alpha_{1} / \alpha_{2}, 1\right)}{\alpha_{2}^{2}}=\frac{\gamma^{*}\left(1, \alpha_{2} / \alpha_{1}\right)}{\alpha_{1}^{2}} .
$$

Proof: By direct calculation, the pdf of the LLR channel estimate corresponding to the threshold is

$$
\begin{aligned}
p_{\Lambda_{0}^{*}\left(\alpha_{1}, \alpha_{2}\right)} & =\frac{1}{2} \mathcal{N}\left(2 \alpha_{1}^{2} \gamma^{*}, 4 \alpha_{1}^{2} \gamma^{*}\right)+\frac{1}{2} \mathcal{N}\left(2 \alpha_{2}^{2} \gamma^{*}, 4 \alpha_{2}^{2} \gamma^{*}\right) \\
& =\frac{1}{2 \alpha_{1}^{2}} \mathcal{N}\left(2 \gamma^{*}, 4 \gamma^{*}\right)+\frac{1}{2 \alpha_{1}^{2}} \mathcal{N}\left(2 \frac{\alpha_{2}^{2}}{\alpha_{1}^{2}} \gamma^{*}, 4 \frac{\alpha_{2}^{2}}{\alpha_{1}^{2}} \gamma^{*}\right) \\
& =\frac{p_{\Lambda_{0}^{*}\left(1, \alpha_{2} / \alpha_{1}\right)}}{\alpha_{1}^{2}} .
\end{aligned}
$$

Similarly, $p_{\Lambda_{0}^{*}\left(\alpha_{1}, \alpha_{2}\right)}=p_{\Lambda_{0}^{*}\left(\alpha_{1} / \alpha_{2}\right)} / \alpha_{2}^{2}$ by symmetry.

This lemma shows that, to obtain the iterative decoding threshold for any pair of fading gains $\left(\alpha_{1}, \alpha_{2}\right)$, it is sufficient to compute the single-argument function $\gamma^{*}(1, \alpha)$, where $\alpha \triangleq \alpha_{2} / \alpha_{1}, \alpha \in R^{+}$. For notational simplicity, we write $\gamma^{*}(\alpha)$ in lieu of $\gamma^{*}(1, \alpha)$.

\section{Numerical Approximation of $\gamma^{*}(\alpha)$}

Extensive numerical simulations have consistently shown a behavior of $\gamma^{*}(\alpha)$ for random codes which differs considerably from that associated with root codes with the same $\lambda(x)$ and $\rho(x)$. The approximations $\widehat{\gamma}_{\text {rand }}^{*}(\alpha)$ and $\widehat{\gamma}_{\text {root }}^{*}(\alpha)$ that follow were seen to match well the numerical results.

For the $(\lambda, \rho)$ random LDPC ensemble,

$$
\widehat{\gamma}_{\text {rand }}^{*}(\alpha)=\frac{a(\alpha)}{\alpha^{2}}+\frac{b(\alpha)}{\alpha}+a(\alpha),
$$

where

$$
\begin{aligned}
& a(\alpha) \triangleq K_{a} e^{-\tau_{a} \alpha} \\
& b(\alpha) \triangleq K_{b}\left(1-e^{-\tau_{b} \alpha}\right)
\end{aligned}
$$

for some constants $K_{a}, K_{b}, \tau_{a}$, and $\tau_{b}$ depending on the code ensemble. Also, for the $(\lambda, \rho)$ root-LDPC ensemble,

$$
\widehat{\gamma}_{\text {root }}^{*}(\alpha)=\frac{c(\alpha)}{\alpha}
$$

with

$$
c(\alpha) \triangleq K_{a}\left(1-e^{-\tau_{a} \alpha}\right)+K_{b} e^{-\tau_{b} \alpha} .
$$

The above approximations are remarkably close to the numerical results obtained for all the distributions $\lambda(x)$ and $\rho(x)$ we used in simulations. Moreover, they capture the difference in diversity for random and root ensembles, and in addition the root-code boundary is seen to approach outage capacity better than its random-code counterpart.

As an example, Figs. 1 and 2 compare simulation and approximation results for random and Root $\left(x^{2}, x^{5}\right)$ LDPC codes, respectively. In both cases, a very good match is observed between numerical results and approximations. The constants estimated are $K_{a}=10^{0.11}, K_{b}=10^{0.65}, \tau_{a}=18$, and $\tau_{b}=18$.

We claim that (3) and (4) give a very accurate approximation of $\gamma(\alpha)$ for random and root LDPC codes respectively. Note 


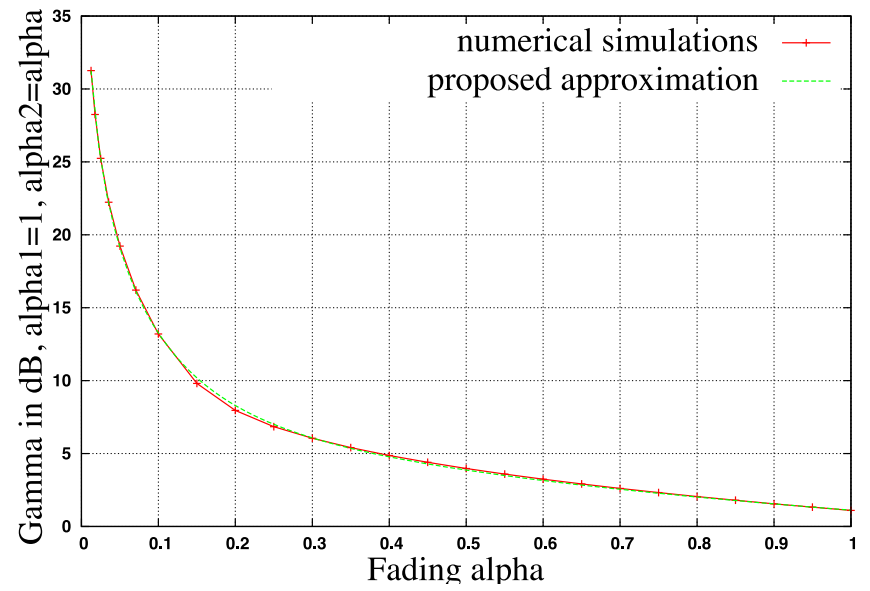

Fig. 1. Approximation $\widehat{\gamma}_{\text {rand }}^{*}(\alpha)$ (dashed line) compared with $\gamma_{\text {rand }}^{*}(\alpha)$ (continuous line) obtained by simulations for the $\left(x^{2}, x^{5}\right)$ random LDPC ensemble.

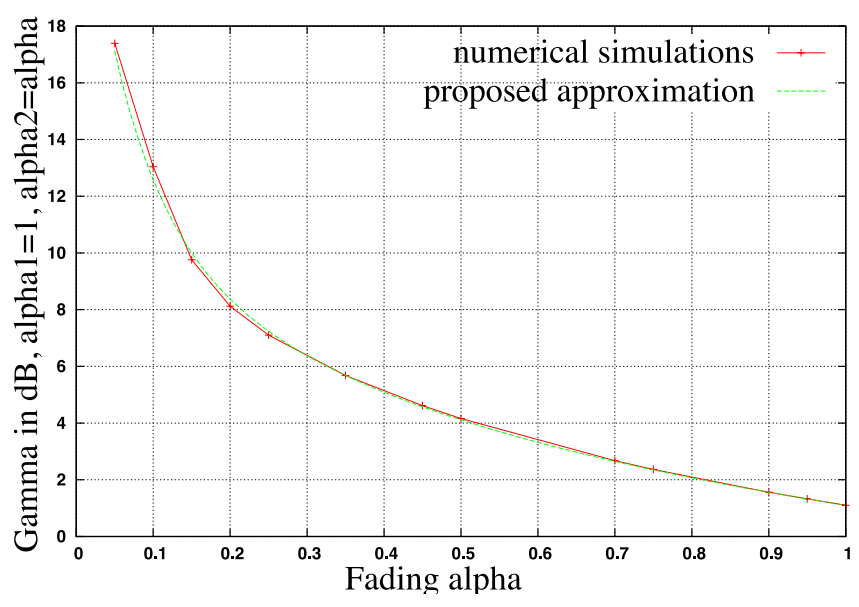

Fig. 2. Approximation $\widehat{\gamma}_{\text {root }}^{*}(\alpha)$ (dashed line) compared with $\gamma_{\text {root }}^{*}(\alpha)$ (continuous) obtained by simulations for the $\left(x^{2}, x^{5}\right)$ root-LDPC ensemble.

that to obtain the approximation $\gamma(\alpha)$ for all $\alpha$ 's, one needs to simulate at most four points for random ensembles, as there are four unknowns to be estimated. For root-LDPC ensembles the maximum nuimber of points is 2 (two unknown parameters in (4)).

\section{Analytical Approximation of $\gamma_{\text {root }}^{*}\left(\alpha_{1}, \alpha_{2}\right)$}

In this section we develop an analytic approximation to the iterative-decoding threshold curve. This is based on the assumption that the behavior of $\gamma_{\text {root }}^{*}\left(\alpha_{1}, \alpha_{2}\right)$ in the high SNR regime is similar to the behavior of the outage boundary, as derived from the outage capacity of the corresponding blockfading channel. An approximation to the outage boundary is determined below.

\section{A. Approximation of the Outage Boundary}

We have an outage whenever $\alpha_{1}^{2}$ and $\alpha_{2}^{2}$ are such that the mutual information between channel input and output is lower than the code rate. Under our assumption of rate $1 / 2$, an outage occurs when $\alpha_{1}$ and $\alpha_{2}$ are such that

$$
\mathbb{E}_{X} \log _{2}\left(1+e^{-2 \alpha_{1}^{2} X}\right)+\mathbb{E}_{X} \log _{2}\left(1+e^{-2 \alpha_{2}^{2} X}\right)>1
$$

where $X$ is the random variable related to the instantaneous channel SNR, $X \sim \mathcal{N}(\gamma, \gamma)$ [3]. Defining

$$
g(\alpha) \triangleq \mathbb{E}_{X} \log _{2}\left(1+e^{-2 \alpha^{2} X}\right)
$$

the condition for an outage becomes

$$
g\left(\alpha_{1}\right)+g\left(\alpha_{2}\right)>1
$$

Explicitly, the function $g(\cdot)$ has the general form

$$
g(\alpha)=\frac{1}{\sqrt{2 \pi \gamma}} \int_{-\infty}^{\infty} \log _{2}\left(1+e^{-2 \alpha^{2} x}\right) e^{-(x-\gamma)^{2} / \gamma} d x
$$

A good approximation to (7) comes from the observation that the integrand function has a maximum around $x=0.5$, a value which does not change much for different values of $\gamma$. Thus,

$$
g(\alpha) \approx \log _{2}\left(1+e^{-\alpha^{2} \gamma}\right)
$$

Under the approximation above, the outage boundary equation is specified by

$$
\log _{2}\left(1+e^{-\alpha_{1}^{2} \gamma}\right)+\log _{2}\left(1+e^{-\alpha_{2}^{2} \gamma}\right)=1
$$

and, hence,

$$
\alpha_{2}^{2}=\frac{1}{\gamma} \ln \frac{1+e^{-\alpha_{1}^{2} \gamma}}{1-e^{-\alpha_{1}^{2} \gamma}}
$$

Using the series expansion $[4,1.513 .1]$, we obtain the outage boundary for large $\alpha_{1}$ :

$$
\alpha_{2}^{2}=\frac{2}{\gamma} e^{-\alpha_{1}^{2} \gamma}
$$

Similarly, for large $\alpha_{2}^{2}$,

$$
\alpha_{1}^{2}=\frac{2}{\gamma} e^{-\alpha_{2}^{2} \gamma}
$$

To verify that the approximation found captures well the behavior of the outage boundary, we compute the outage probability using approximation (8):

$$
\begin{aligned}
P_{\mathrm{out}} & \approx \mathbb{P}\left(\log _{2}\left(1+e^{-\alpha_{1}^{2} \gamma}\right)\left(1+e^{-\alpha_{2}^{2} \gamma}\right)>1\right) \\
& =\mathbb{P}\left(\left(1+e^{-\alpha_{1}^{2} \gamma}\right)\left(1+e^{-\alpha_{2}^{2} \gamma}\right)>2\right) \\
& =\mathbb{P}\left(1+e^{-\alpha_{1}^{2} \gamma}>\frac{2}{1+e^{-\alpha_{2}^{2} \gamma}}\right) \\
& =\mathbb{P}\left(\alpha_{1}^{2}<\frac{1}{\gamma} \ln \frac{1+e^{-\alpha_{2}^{2} \gamma}}{1-e^{-\alpha_{2}^{2} \gamma}}\right)
\end{aligned}
$$

Under the assumption of Rayleigh fading, $\alpha_{1}^{2}$ has an exponential density, and hence we may use the approximation, valid for small $x$,

$$
\mathbb{P}\left(\alpha_{1}^{2}<x\right) \approx x .
$$

which yields

$$
P_{\mathrm{out}} \approx \frac{1}{\gamma} \mathbb{E}\left[\ln \frac{1+e^{-\alpha_{2}^{2} \gamma}}{1-e^{-\alpha_{2}^{2} \gamma}}\right]
$$


Using the fact that $\ln \frac{1+e^{-\alpha_{2}^{2} \gamma}}{1-e^{-\alpha_{2}^{2} \gamma}} \approx 2 e^{-\alpha_{2}^{2} \gamma}$, we obtain

$$
\begin{aligned}
P_{\text {out }} & \approx \frac{2}{\gamma} \mathbb{E}\left[e^{-\alpha_{2}^{2} \gamma}\right] \\
& =\frac{2}{\gamma} \int_{0}^{\infty} e^{-x(\gamma+1)} d x \\
& =\frac{2}{\gamma(\gamma+1)} .
\end{aligned}
$$

Finally, for high SNR (large $\gamma)$

$$
P_{\text {out }} \approx \frac{2}{\gamma^{2}}
$$

which is consistent with the result given in [3].

\section{B. $\gamma^{*}\left(\alpha_{1}, \alpha_{2}\right)$ for Root-LDPC Codes at High SNR}

Here we derive an approximation of the threshold curve for Root-LDPC ensembles, assuming that its behavior at high SNRs is the same as the one of the outage boundary. We have the following theorem:

Theorem 1: Consider a $(\lambda, \rho)$ Root-LDPC ensemble. Then

$$
\gamma_{\text {root }}^{*}\left(\alpha_{1}, \alpha_{2}\right) \approx \frac{1}{\alpha_{1}^{2}} W\left(2 \alpha_{1}^{2} / \alpha_{2}^{2}\right)
$$

or, equivalently,

$$
\gamma_{\text {root }}^{*}\left(\alpha_{1}, \alpha_{2}\right) \approx \frac{1}{\alpha_{2}^{2}} W\left(2 \alpha_{2}^{2} / \alpha_{1}^{2}\right),
$$

where $W(\cdot)$ is the Lambert function [5].

Proof: We are interested in studying the behavior of the function

$$
\gamma=\gamma_{\text {root }}^{*}\left(\alpha_{1}, \alpha_{2}\right)
$$

where $\gamma, \alpha_{1}^{2}$, and $\alpha_{2}^{2}$ are related by the outage boundary condition

$$
\mathbb{E}_{X} \log _{2}\left(1+e^{-2 \alpha_{1}^{2} X}\right)+\mathbb{E}_{X} \log _{2}\left(1+e^{-2 \alpha_{2}^{2} X}\right)=1
$$

with $X \sim \mathcal{N}(\gamma, \gamma)$. As before, we may approximate (13) with

$$
\log _{2}\left(1+e^{-\alpha_{1}^{2} \gamma}\right)+\log _{2}\left(1+e^{-\alpha_{2}^{2} \gamma}\right)=1
$$

From (14), we can see that the function $g(\cdot)$ satisfies

$$
\gamma_{\text {root }}^{*}\left(\alpha_{1}, \alpha_{2}\right)=\frac{1}{\alpha_{1}^{2}} \gamma_{\text {root }}^{*}\left(1, \alpha_{2} / \alpha_{1}\right) \triangleq \frac{1}{\alpha_{1}^{2}} \gamma_{\text {root }}^{*}\left(\alpha_{2} / \alpha_{1}\right)
$$

which is consistent with Lemma 1 . So, our goal is tantamount to studying the single-argument function $\beta(\gamma)$, where

$$
\left(1+e^{-\gamma}\right)\left(1+e^{-\beta \gamma}\right)=2
$$

and have defined $\beta \triangleq \alpha_{2}^{2} / \alpha_{1}^{2}$. Given the symmetry of the problem with respect to $\alpha_{1}$ and $\alpha_{2}$, we may constrain ourselves to the consideration of either $\alpha_{2} \leq \alpha_{1}$ or $\alpha_{1} \leq \alpha_{2}$. Thus, $\beta \in[0,1]$ corresponds to $\alpha_{2} \leq \alpha_{1}$, while $\beta \in[1, \infty)$ corresponds to $\alpha_{1} \leq \alpha_{2}$.

We have the pair of values $\beta(\infty)=0$ and $\beta(0)=\infty$, and the expression

$$
\beta=\frac{1}{\gamma} \ln \frac{1+e^{-\gamma}}{1-e^{-\gamma}}
$$

Using again the series expansion $[4,1.513 .1]$

$$
\ln \frac{1+x}{1-x}=2 \sum_{k=1}^{\infty} \frac{1}{2 k-1} x^{2 k-1}, \quad|x|<1
$$

we have

$$
\beta=\frac{2}{\gamma} \sum_{k=1}^{\infty} \frac{1}{2 k-1} e^{-(2 k-1) \gamma}=\frac{2}{\gamma}\left(e^{-\gamma}+\frac{1}{3} e^{-3 \gamma}+\cdots\right)
$$

and we can finally obtain the sought function $\gamma(\beta)$ by inverting (16).

This can be done by observing that

$$
\beta=\frac{2}{\gamma} e^{-\gamma}
$$

is equivalent to

$$
\frac{2}{\beta}=\gamma e^{\gamma}
$$

Inversion of this function yields

$$
\gamma=W(2 / \beta)
$$

so that, finally,

$$
\gamma_{\text {root }}^{*}\left(\alpha_{1}, \alpha_{2}\right)=\frac{1}{\alpha_{1}^{2}} \gamma_{\text {root }}^{*}\left(\alpha_{2} / \alpha_{1}\right) \approx \frac{1}{\alpha_{1}^{2}} W\left(2 \alpha_{1}^{2} / \alpha_{2}^{2}\right) .
$$

Comparing the approximation of Theorem 1 against numerical results, we can see that indeed it gives a good match in the high-SNR region. However, the approximation of the threshold function, derived through the outage boundary approach, is not satisfactory at intermediate-to-low SNRs, as it does not depend on the ensemble parameters.

\section{DISCUSSION}

In this paper we have presented estimates of the iterative threshold behavior of a sparse-graph code ensemble for the transmission over a nonergodic block-fading Rayleigh channel with two blocks affected by two independent Rayleighdistributed fading gains. The first approximation comes from the interpolation of numerical data, and allows one to obtain a close estimate of the whole threshold behavior by simulating only several points of the threshold curve, for both random LDPC codes and rate-1/2 Root-LDPC codes, based on degree distributions $\lambda(x)$ and $\rho(x)$. The second approximation concerns rate-1/2 $(\lambda(x), \rho(x))$-Root-LDPC codes, it is analytic, and works well in the high-SNR regime.

\section{REFERENCES}

[1] J. Boutros, A. Guillén i Fàbregas, E. Biglieri, and G. Zémor, "Lowdensity parity-check codes for nonergodic block-fading channels," 2007, submitted to the IEEE Trans. Inform. Theory.

[2] X. Jin, A. Eckford, and T. Fuja, "Design of good low-density parity-check codes for block fading channels," in MILCOM'04, October 2004.

[3] J. Boutros, "Diversity and coding gain evolution in graph codes," in ITA'09, San Diego, CA, February 2009.

[4] I. S. Gradshteyn and I. M. Ryzhik, Table of Integrals, Series, and Products, 7th ed. Amsterdam, The Netherlands: Academic Press, 2007.

[5] R. M. Corless, G. H. Gonnet, D. E. G. Hare, D. J. Jeffrey, and D. E. Knuth, "On the Lambert W function," Advances in Computational Mathematics, vol. 5, pp. 329-359, 1996. 